\title{
Weniger Exazerbationen durch extrafeine ICS / LABA-Fixkombination
}

Die kleinen Atemwege sind wesentlich von der Entzündung bei chronisch obstruktiver Bronchitis (COPD) betroffen. Für die extrafeine Fixkombination ${ }^{1}$ aus dem inhalativen Kortikoid (ICS) Beclometason und dem langwirksamem Beta-2-Mimetikum (LABA) Formoterol wurde eine hohe und gleichmäßige Deposition bis in die Lungenperipherie szintigraphisch nachgewiesen. Im Rahmen des diesjährigen ERS-Kongresses wurde die Wichtigkeit der Therapie der kleinen Atemwege diskutiert.

Ein wichtiges Ziel der COPD-Therapie ist, neben der Verbesserung der Symptomatik, die Reduktion des Exazerbationsrisikos. Als klinisch vorteilhaft hat sich in Studien die inhalative Therapie mit der extrafeinen ICS/LABA-Fixkombination aus Beclometason und Formoterol erwiesen. Es wurde eine gute Deposition in den großen und kleinen Atemwegen nachgewiesen.
Die Bedeutung der Eosinophilen im Hinblick auf die Wirkung einer ICS/LABATherapie wird gegenwärtig kontrovers diskutiert. Für die extrafeine ICS/LABAFixkombination aus Beclometason und Formoterol wurde eine konstant niedrige Exazerbationsrate - unabhängig von der Zahl der Blut-Eosinophilen - nachgewie$\operatorname{sen}^{2}$. Zu diesem Ergebnis kommt eine Post hoc-Analyse der Daten aus der randomisierten Doppelblindstudie FORWARD, in der Patienten mit schwerer COPD über einen Zeitraum von 48 Wochen entweder die extrafeine Fixkombination Beclometason/Formoterol oder eine FormoterolMonotherapie erhielten. Bezogen auf die Studiengesamtpopulation der FORWARDStudie konnte unter Beclometason/Formoterol die Exazerbationsrate im Vergleich zur Formoterol-Monotherapie deutlich um $28 \%$ reduziert werden.

Es wurde weiterhin untersucht, inwieweit die Therapieeffekte von unterschiedli- chen Blut-Eosinophilen-Werten abhängig sind. Dafür wurden die Daten von 1184 Patienten ausgewertet. Anhand der BlutEosinophilen-Zahlen erfolgte eine Einteilung in 4 Quartile mit jeweils gleicher Patientenzahl. In allen 4 EosinophilenQuartilen zeigten die Patienten unter der extrafeinen ICS/LABA-Fixkombination konstant niedrige Exazerbationsraten. Unter der LABA-Monotherapie nahm hingegen die Exazerbationshäufigkeit mit steigenden Eosinophilenwerten zu. Diese Ergebnisse wurden durch einen vergleichbaren Trend in Bezug auf die Zeit bis zur 1. Exazerbation untermauert. In einem prädiktiven Modell wurde auf Basis dieser Studiendaten das Risiko zukünftiger Exazerbationen in Abhängigkeit von den Eosinophilenwerten abgebildet. Bei Patienten unter extrafeinem Beclometason / Formoterol wurde das Exazerbationsrisiko im Gegensatz zu Patienten unter Formoterol nicht durch die Zahl der Blut-Eosinophilen beeinflusst.

\section{Nach einer Pressemitteilung (Chiesi)}

${ }^{1}$ Foster $^{\circledR}$, Chiesi GmbH, Hamburg

2 Siddiqui SH et al. AJRCCM 2015; 192: 523-525

Serotyp-bedingte CAP auftraten $(p=0,0006)$. Darüber hinaus war bei den geimpften Teilnehmern das Auftreten einer nicht bakteriämischen/nicht invasiven Impfstoff-Serotyp-bedingten CAP um $45 \%(\mathrm{p}=0,0067)$ und das einer ImpfstoffSerotyp-bedingten IPD um 75\% im Vergleich zur Kontrollgruppe reduziert $(\mathrm{p}=0,0005)$.

Die PCV13-Impfung immunisiert gegen 13 Serotypen von Streptococcus pneumoniae. Die Kosten der Impfung werden durch die Krankenkassen für Erwachsene im Alter von mindestens 60 Jahren sowie bei Patienten mit chronischen Grunderkrankungen in jedem Alter übernommen.

\section{Monika Walter, München}

Menschen das Auftreten einer Pneumokokken-Pneumonie verhindern $\mathrm{kann}^{3}$ : Die doppelblinde, randomisierte, placebokontrollierte Studie schloss rund 85000 Personen ab 65 Jahre ein. Untersucht wurde die Wirksamkeit von PCV13 zur Prävention einer ersten Episode einer Impfstoff-Serotyp-spezifischen Erkrankung (CAP, nicht bakteriämische/ nicht invasive CAP und IPD). Die Auswertung demonstrierte, dass bei mit PCV13 geimpften Studienteilnehmern im Vergleich zur Placebogruppe um 45,56\% weniger Impfstoff-

\footnotetext{
'Fachpressegespräch „Prävention von Pneumokokken-Pneumonie: Die Bedeutung der Impfung gegen Pneumokokken und die Besonderheiten des Konjugatimpfstoffs Prevenar $13^{\circledR}$, Oktober 2015, München, Veranstalter: Pfizer Deutschland $\mathrm{GmbH}$

2 Prevenar $13^{\circledR}$, Pfizer Deutschland $\mathrm{GmbH}$, Berlin

${ }^{3}$ Bonten MJM et al. N Engl J Med 2015; 372: 1114-1125
} 